\title{
Phase space decomposition for phase noise and synchronization analysis of planar nonlinear oscillators
}

\author{
Michele Bonnin, Fernando Corinto, Marco Gilli
}

May 14, 2019

\begin{abstract}
Synchronization phenomena, frequency shift and phase noise are often limiting key factors in the performances of oscillators. The perturbation projection method allows to characterize how the oscillator's output is modified by these disturbances. In this brief we discuss the appropriate decomposition of perturbations for synchronization and phase noise analysis of planar nonlinear oscillators. We derive analytical formulas for the vectors spanning the directions along which the perturbations have to be projected. We also discuss the implications of this decomposition in control theory and to what extent a simple orthogonal projection is correct.
\end{abstract}

\section{Introduction}

Oscillators are ubiquitous in modern electronic and optical devices. For instance, in digital systems they are responsible to give a reference signal to synchronize operations. In radio communication systems they are used for frequency and amplitude modulations to convey information.

An ideal oscillator would exhibit a perfectly localized spectrum at the desired frequency. However, the output of actual oscillators is always corrupted by external disturbances, internal noise source, thermal noise, and interaction with other oscillators. As a consequence the power spectra of practical oscillators exhibit both a shift of its peaks and linewidth broadening. These phenomena, often referred to as frequency shift and phase noise, are key performance limiting factors in electronic systems. Characterizing how perturbations affect the performances of oscillators is therefore a problem of both theoretical and practical paramount importance, which has received lot of attention since the seminal work [1].

${ }^{*}$ The authors are with the Department of Electronics and Telecommunications, Politecnico di Torino, Turin, Italy (e-mail: michele.bonnin@polito.it). 
The traditional approach is to linearize the system about the unperturbed solution, assuming that the resultant deviation are small [2, 3. However it turns out that in several situations the small deviation assumption becomes invalid and the linearized perturbation analysis is inconsistent [4, 5. To overcome this limitation nonlinear analysis methods, based on the decomposition of the perturbation into orthogonal components has been proposed [4, 5]. The method has later been improved with the introduction of a non orthogonal decomposition, leading to the so called perturbation projection method [6, 7].

The perturbation projection method has been used to study injection locking [8, 9, pulling effects [10, synchronization phenomena [11, and power spectra of noisy oscillators [12, 7, 13]. Different numerical schemes have been proposed 7, 12, 8, 14, 15, to find the proper decomposition of the perturbation, that has to be projected onto two complementary subspaces. It turns out that the bases of the two spaces are related to Floquet's eigenvectors, and their determination requires several numerical integrations of both linear and nonlinear differential problems, a potentially burdensome task [6, 16, 15].

This brief discusses the appropriate phase space decomposition for planar nonlinear oscillators. The main contribution of the paper is the derivation of analytical formulas for the vectors spanning the subspaces along which the perturbations has to be decomposed. Using a classical result on the integration of planar autonomous differential equations we derive these formulas, that allow to write a nonlinear differential equation for the time evolution of the oscillator's phase deviation in a closed form. This equation is the starting point to investigate synchronization phenomena, frequency shift and phase noise in weakly perturbed oscillators. We also discuss some implication of the decomposition in control theory and to what extent the old orthogonal decomposition is correct.

\section{Phase space decomposition of nonlinear oscil- lations}

We consider nonlinear oscillators subject to external perturbations described by the ODE

$$
\dot{\mathbf{x}}(t)=\mathbf{f}(\mathbf{x}(t))+\varepsilon \mathbf{g}(\mathbf{x}(t), t)
$$

where $\mathbf{x}: \mathbb{R} \mapsto \mathbb{R}^{n}$ is the state of the oscillator, $\mathbf{f}: \mathbb{R}^{n} \mapsto \mathbb{R}^{n}$ describes the oscillator's internal dynamics, $\mathrm{g}: \mathbb{R}^{n} \times \mathbb{R} \mapsto \mathbb{R}^{n}$ defines the perturbation and $\varepsilon \ll 1$ measures the strength of the perturbation. In absence of the perturbation, e.g. for $\varepsilon=0$, the oscillator exhibits an asymptotically stable $T$-periodic limit cycle $\gamma$

$$
\left\{\begin{array}{l}
\dot{\mathbf{x}}_{0}(t)=\mathbf{f}\left(\mathbf{x}_{0}(t)\right) \\
\mathbf{x}_{0}(t)=\mathbf{x}_{0}(t+T)
\end{array}\right.
$$

The ideal framework to investigate phase noise effects, phase locking and synchronization phenomena in (11) are phase models [6. Phase models are based on the idea to decompose the perturbation into two components, one tangent 
and one transversal, but not perpendicular to, the unperturbed limit cycle. The effect of the tangential component is to induce a phase shift in the oscillation, leaving the amplitude unchanged. The effect of the transversal component, sometimes called oblique component, is to modify the amplitude, without affecting the phase of the oscillation.

Under the effect of the perturbation, the response of the oscillator becomes

$$
\mathbf{x}(t)=\mathbf{x}_{0}(t+\psi(t))+\mathbf{z}(t)
$$

where $\mathbf{z}(t)$ describes a small perturbation of the amplitude which decays exponentially fast, while $\psi(t)$ is a phase shift induced by the injected signal. It can be shown [6] that $\psi(t)$ is the solution of the nonlinear phase deviation equation

$$
\dot{\psi}(t)=\mathbf{v}_{1}^{T}(t+\psi(t)) \mathbf{g}\left(\mathbf{x}_{0}(t+\psi(t)), t\right)
$$

The vector $\mathbf{v}_{1}(t)$ is the unique non trivial $T$-periodic solution of the adjoint problem

$$
\dot{\mathbf{y}}(t)=-\mathbf{D f}\left(\mathbf{x}_{0}(t)\right)^{T} \mathbf{y}(t)
$$

satisfying the normalization condition

$$
\mathbf{y}^{T}(t) \mathbf{f}\left(\mathbf{x}_{0}(t)\right)=1 \quad \forall t \in \mathbb{R}^{+} .
$$

Noisy signals are modelled by the perturbation

$$
\mathbf{g}(\mathbf{x}(t), t)=\mathbf{G}(\mathbf{x}(t)) \Gamma(t)
$$

where $\mathbf{G}: \mathbb{R}^{n} \mapsto \mathbb{R}^{n \times m}$ is a state dependent matrix and $\Gamma: \mathbb{R} \mapsto \mathbb{R}^{m}$ is the vector of noise components. The corresponding phase deviation equation (4) is a stochastic differential equation that requires a probabilistic treatment. The time evolution for the probability density function $p(\psi, t)$ is given by the Fokker-Planck equation [6]

$$
\begin{aligned}
\frac{\partial p(\psi, t)}{\partial t}= & -\frac{\partial}{\partial \psi}\left(\mathbf{v}(t+\psi) \frac{\partial \mathbf{v}(t+\psi)^{T}}{\partial \psi} p(\psi, t)\right) \\
& +\frac{\partial^{2}}{\partial \psi^{2}}\left(\mathbf{v}(t+\psi)^{T} \mathbf{v}(t+\psi) p(\psi, t)\right)
\end{aligned}
$$

where $\mathbf{v}(t)^{T}=\mathbf{v}_{1}(t)^{T} \mathbf{G}(\mathbf{x}(t))$.

Both eq. (5) and (8) show that an analytical expression for the vector $\mathbf{v}_{1}(t)$ is necessary to write down the phase deviation equation and the Fokker-Planck equation in a closed form. Unfortunately, in most of situations this vector can only be determined numerically. In the next sections we shall derive an analytical formula for the vector $\mathbf{v}_{1}$ in terms of the unperturbed vector field $\mathbf{f}(\cdot)$ and the trajectory $\mathbf{x}_{0}(t)$ which holds for any planar oscillator. 


\section{Diliberto's theorem}

The Diliberto's theorem is a classical result on the integration of planar homogeneous linear differential equations in terms of geometrical quantities along a given trajectory of the system. For a given vector field $\mathbf{f}=\left(f_{1}, f_{2}\right)^{T}$, we introduce the perpendicular vector field $\mathbf{f}^{\perp}=\left(f_{2},-f_{1}\right)^{T}$, and we denote the jacobian matrix by $\mathbf{A}(t)=\mathbf{D} \mathbf{f}\left(\mathbf{x}_{0}(t)\right)$.

Theorem 1 (Diliberto's theorem [17]) Let $\mathbf{f}\left(\mathbf{x}_{0}(t)\right)$ be a solution of the variational equation

$$
\dot{\mathbf{x}}(t)=\mathbf{A}(t) \mathbf{x}(t)
$$

Then the fundamental matrix solution has the form

$$
\widetilde{\mathbf{X}}(t)=\left[\begin{array}{ll}
\mathbf{f}\left(\mathbf{x}_{0}(t)\right) & a(t) \mathbf{f}\left(\mathbf{x}_{0}(t)\right)+\frac{b(t)}{\left\|\mathbf{f}\left(\mathbf{x}_{0}(t)\right)\right\|^{2}} \mathbf{f}^{\perp}\left(\mathbf{x}_{0}(t)\right)
\end{array}\right]
$$

where $\|\mathbf{f}(\cdot)\|$ denotes the usual $L_{2}$ norm, $a(t)$ and $b(t)$ are given by

$$
\begin{aligned}
& b(t)=e^{\int_{0}^{t} \nabla \cdot \mathbf{f}\left(\mathbf{x}_{0}(s)\right) d s} \\
& a(t)=\int_{0}^{t} \frac{\mathbf{f}^{T}\left(\mathbf{x}_{0}(s)\right)\left(\mathbf{A}(s)+\mathbf{A}(s)^{T}\right) \mathbf{f}^{\perp}\left(\mathbf{x}_{0}(s)\right)}{\left\|\mathbf{f}\left(\mathbf{x}_{0}(s)\right)\right\|^{4}} b(s) d s
\end{aligned}
$$

and $\nabla \cdot$ is the divergence operator.

See [17 for a proof of the theorem.

The Diliberto's theorem has important applications in the analysis of planar nonlinear oscillations. If $\mathbf{A}(t)=\mathbf{D} \mathbf{f}\left(\mathbf{x}_{0}(t)\right)$ is the jacobian matrix of system (11) in absence of perturbation, then eq. (9) determines the stability of the limit cycle. It is trivial to verify that if $\mathbf{x}_{0}(t)$ is a solution of eq. (11) for $\varepsilon=0$, then $\mathbf{f}\left(\mathbf{x}_{0}(t)\right)$ solves eq. (9). Thus one can make use of Diliberto's theorem to find the fundamental matrix solution of the variational equation (9) and to compute the Floquet's multipliers, which determine the stability of the limit cycle.

At each point $\mathbf{x} \in \gamma$, the unperturbed oscillator's (described by $\dot{\mathbf{x}}(t)=$ $\mathbf{f}(\mathbf{x}(t)))$ stable manifold can be decomposed into two complementary linear spaces, the space $T M_{\mathbf{x}}$ tangent to the unperturbed limit cycle at $\mathbf{x}$, and the space $T I_{\mathbf{x}}$ tangent to the isochron at $\mathbf{x}\left[15\right.$. Let $\mathbf{x}_{i}(t), i=0,1, \ldots$ be trajectories (solutions) of the unperturbed system with initial condition $\mathbf{x}_{i}$, with $\mathbf{x}_{0} \in \boldsymbol{\gamma}$. The isochron based at $\mathbf{x}_{0}$ is defined as the set of all initial conditions $\mathbf{x}_{i}$ such that the trajectories $\mathbf{x}_{i}(t)$ asymptotically converge to $\mathbf{x}_{0}(t)$ on the limit cycle (see figure 2). Extending the definition to all points $\mathbf{x} \in \gamma$ leads to the tangent bundles $T M$ and $T I$. The tangent bundles $T M$ and $T I$ are spanned by the vectors $\mathbf{u}_{1}(t)$ and $\mathbf{u}_{2}(t)$, respectively, which are the Floquet's eigenvectors of the variational equation (9) [6, 15] 1 . In general finding the Floquet's eigenvectors

\footnotetext{
${ }^{1}$ For a $n$-order oscillator, $T I_{\mathbf{x}}$ is spanned by $\left\{\mathbf{u}_{2}(t), \ldots, \mathbf{u}_{n}(t)\right\}$.
} 


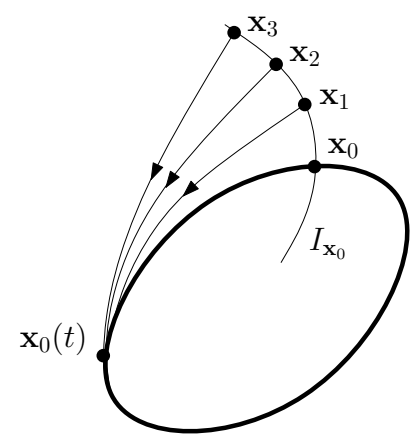

Figure 1: Definition of an isochron.

requires several numerical integrations $\underline{6}$, but in the planar case, we shall show that using Diliberto's theorem analytical formulas for $\mathbf{u}_{1}(t)$ and $\mathbf{u}_{2}(t)$ can be derived 2 .

\section{Phase space decomposition using Diliberto's theorem}

In this section we derive analytical formulas, in terms of the unperturbed limit cycle, for the vectors spanning the tangent bundles $T M$ and $T I$.

Theorem 2 The Floquet's eigenvectors $\mathbf{u}_{1}(t)$ and $\mathbf{u}_{2}(t)$, spanning the tangent bundles $T M$ and $T I$ are given by

$$
\begin{aligned}
& \mathbf{u}_{1}(t)=\mathbf{f}\left(\mathbf{x}_{0}(t)\right) \\
& \mathbf{u}_{2}(t)=e^{-\mu_{2} t}\left(\alpha(t) \mathbf{f}\left(\mathbf{x}_{0}(t)\right)+\beta(t) \mathbf{f}^{\perp}\left(\mathbf{x}_{0}(t)\right)\right)
\end{aligned}
$$

where $\mu_{2}$ is the second Floquet's exponent and

$$
\begin{aligned}
\alpha(t) & =\frac{a(T)}{b(T)-1}+a(t) \\
\beta(t) & =\frac{b(t)}{\left\|\mathbf{f}\left(\mathbf{x}_{0}(t)\right)\right\|^{2}}
\end{aligned}
$$

with $a(T)$ and $b(T)$ given by (11) and (12).

Proof: for shorthand of notation, we introduce the vector field $\mathbf{F}: \mathbb{R} \mapsto \mathbb{R}^{n}$ defined by $\mathbf{F}=\mathbf{f} \circ \mathbf{x}_{0}$, e.g. $\mathbf{F}(t)=\mathbf{f}\left(\mathbf{x}_{0}(t)\right)$.

\footnotetext{
${ }^{2}$ The application to higher order system is nontrivial because a full generalization of Diliberto's theorem to higher dimensions is still an open issue.
} 
It is straightforward to obtain the inverse of (10)

$$
\widetilde{\mathbf{X}}^{-1}(t)=\frac{1}{b(t)}\left[\begin{array}{c}
-a(t) \mathbf{F}^{\perp}(t)^{T}+\frac{b(t)}{\|\mathbf{F}(t)\|^{2}} \mathbf{F}(t)^{T} \\
\mathbf{F}^{\perp}(t)^{T}
\end{array}\right]
$$

We can use (17) to construct the state transition matrix $\widetilde{\mathbf{X}}(t, 0)=\widetilde{\mathbf{X}}^{-1}(0) \widetilde{\mathbf{X}}(t)$, i.e. the fundamental matrix satisfying $\widetilde{\mathbf{X}}(0)=\mathbb{1}$, where $\mathbb{1}$ is the identity matrix,

$$
\begin{aligned}
& \widetilde{\mathbf{X}}(t, 0)= \\
& {\left[\begin{array}{cl}
\frac{\mathbf{F}(0)^{T}}{\|\mathbf{F}(0)\|^{2}} \mathbf{F}(t) & \frac{\mathbf{F}(0)^{T}}{\|\mathbf{F}(0)\|^{2}}\left(a(t) \mathbf{F}(t)+\frac{b(t)}{\|\mathbf{F}(t)\|^{2}} \mathbf{F}^{\perp}(t)\right) \\
\mathbf{F}^{\perp}(0) \mathbf{F}(t) & \mathbf{F}^{\perp}(0)^{T}\left(a(t) \mathbf{F}(t)+\frac{b(t)}{\|\mathbf{F}(t)\|^{2}} \mathbf{F}^{\perp}(t)\right)
\end{array}\right]}
\end{aligned}
$$

Keeping in mind that $\mathbf{F}(T)=\mathbf{F}(0)$ since $\mathbf{f}\left(\mathbf{x}_{0}(T)\right)=\mathbf{f}\left(\mathbf{x}_{0}(0)\right)$, we derive the monodromy matrix

$$
\widetilde{\mathbf{X}}(T, 0)=\left[\begin{array}{ll}
1 & a(T) \\
0 & b(T)
\end{array}\right]
$$

The eigenvalues of the monodromy matrix are the characteristic (Floquet's) multipliers $\lambda_{i}, i=1,2$. They are related to the characteristic exponents $\mu_{i}$ by the relation

$$
\lambda_{i}=e^{\mu_{i} T}
$$

In this case, the multipliers are real and the exponents are 3

$$
\mu_{1}=0 \quad \mu_{2}=\frac{1}{T} \int_{0}^{T} \nabla \cdot \mathbf{f}\left(\mathbf{x}_{0}(s)\right) d s
$$

where eq. (11) has been used.

According to Floquet's theory [6], the state transition matrix can be written in the form

$$
\widetilde{\mathbf{X}}(t, 0)=\widetilde{\mathbf{U}}(t) \exp (\mathbf{D} t) \widetilde{\mathbf{U}}(0)^{-1}
$$

where $\widetilde{\mathbf{U}}(t)$ is a $T$-periodic matrix $\widetilde{\mathbf{U}}(t)=\widetilde{\mathbf{U}}(t+T)$, such that $\widetilde{\mathbf{U}}(0)$ is the matrix of the eigenvectors of the monodromy matrix $\widetilde{\mathbf{X}}(T, 0)$, i.e.

$$
\boldsymbol{\Lambda}=\widetilde{\mathbf{U}}(0)^{-1} \mathbf{X}(T, 0) \widetilde{\mathbf{U}}(0)
$$

with $\boldsymbol{\Lambda}=\operatorname{diag}\left[\lambda_{1}, \lambda_{2}\right]$, and $\mathbf{D}=\operatorname{diag}\left[\mu_{1}, \mu_{2}\right]$.

Computing the eigenvectors of (19) and from (22) we derive

$$
\begin{aligned}
& \widetilde{\mathbf{U}}(t)=\left[\begin{array}{cc}
\frac{\mathbf{F}(0)^{T}}{\|\mathbf{F}(0)\|^{2}} \mathbf{F}(t) & \frac{\mathbf{F}(0)^{T}}{\|\mathbf{F}(0)\|^{2}}\left(\alpha(t) \mathbf{F}(t)+\beta(t) \mathbf{F}^{\perp}(t)\right) \\
\mathbf{F}^{\perp}(0)^{T} \mathbf{F}(t) & \mathbf{F}^{\perp}(0)^{T}\left(\alpha(t) \mathbf{F}(t)+\beta(t) \mathbf{F}^{\perp}(t)\right)
\end{array}\right] \\
& \times \exp (-\mathbf{D} t)
\end{aligned}
$$

\footnotetext{
${ }^{3}$ For $n$-order systems, the Floquet's multipliers are, in general, complex numbers. Therefore one has $\mu_{i}=\frac{1}{T} \ln \left(\left|\lambda_{i}\right|+i\left(\arg \lambda_{i}+2 k \pi\right)\right)$ and an infinite number of characteristic exponents corresponds to the same multiplier.
} 
where

$$
\begin{aligned}
\alpha(t) & =\frac{a(T)}{b(T)-1}+a(t) \\
\beta(t) & =\frac{b(t)}{\|\mathbf{F}(t)\|^{2}}
\end{aligned}
$$

It is well known that the state transition matrix is not unique, because it can be constructed starting from different fundamental matrices, corresponding to different initial conditions. However, all the state transition matrices are similar, that is, if $\mathbf{X}(t, 0)$ and $\widetilde{\mathbf{X}}(t, 0)$ are state transition matrices, then a matrix $\mathbf{C}$ exists such that $\widetilde{\mathbf{X}}(t, 0)=\mathbf{C X}(t, 0) \mathbf{C}^{-1}$. On the one hand we have

$$
\mathbf{X}(t, 0)=\mathbf{U}(t) \exp (\mathbf{D} t) \mathbf{U}(0)^{-1}
$$

and on the other hand

$$
\mathbf{X}(t, 0)=\mathbf{C}^{-1} \widetilde{\mathbf{U}}(t) \exp (\mathbf{D} t) \tilde{\mathbf{U}}(0)^{-1} \mathbf{C}
$$

By comparison we have $\widetilde{\mathbf{U}}(t)=\mathbf{C} \mathbf{U}(t)$, and by looking at (24) we can choose

$$
\mathbf{C}=\left[\begin{array}{c}
\mathbf{F}(0)^{T} \\
\| \mathbf{F}\left(0 \|^{2}\right. \\
\mathbf{F}^{\perp}(0)^{T}
\end{array}\right]
$$

The matrix $\mathbf{U}(t)=\left[\mathbf{u}_{1}(t), \mathbf{u}_{2}(t)\right]$ is given by

$$
\mathbf{U}(t)=\left[\mathbf{F}(t) \quad \alpha(t) \mathbf{F}(t)+\beta(t) \mathbf{F}^{\perp}(t)\right] \exp (-\mathbf{D} t)
$$

and the theorem is proved.

Next we verify that $\mathbf{U}(t)$ is indeed $T$-periodic.

Corollary 1 The matrix $\mathbf{U}(t)$ is T-periodic

Proof: it is obvious that the first column is periodic with period $T$. The second column is periodic if and only if

$$
\left\{\begin{array}{l}
\alpha(t+T)=\alpha(t) e^{\mu_{2} T} \\
\beta(t+T)=\beta(t) e^{\mu_{2} T}
\end{array}\right.
$$

The second condition implies $b(t+T)=b(t) e^{\mu_{2} T}=b(t) b(T)$, which is easily verified by using definition (11), the additivity of integrals and the fact that $\nabla \cdot \mathbf{f}\left(\mathbf{x}_{0}(t)\right)$ is a function of a periodic argument and then it is also periodic.

The first condition leads to $a(t) b(T)=a(t+T)-a(T)$. Let us denote

$$
c(s)=\frac{\mathbf{f}\left(\mathbf{x}_{0}(s)\right)\left(\mathbf{A}(s)+\mathbf{A}(s)^{T}\right) \mathbf{f}^{\perp}\left(\mathbf{x}_{0}(s)\right)}{\left\|\mathbf{f}\left(\mathbf{x}_{0}(s)\right)\right\|^{4}}
$$


obviously $c(s)=c(s+T)$. Using the definition of $a(t)$ given by (12) and the additivity of integrals, the following equality must hold

$$
\int_{0}^{t} c(s) b(s) b(T) d s=\int_{T}^{T+t} c(r) b(r) d r
$$

With the substitution $q=r-T$, using the periodicity of $c(s)$ and the property $b(t+T)=b(t) b(T)$ we obtain an identity.

At each time instant the vectors $\mathbf{u}_{1}(t)$ and $\mathbf{u}_{2}(t)$ are tangent to the unperturbed limit cycle and to the isochron, respectively, at the point $\mathbf{x}_{0}(t)$, thus identifying the tangential and the transversal direction into which the perturbation must be decomposed.

\section{Determination of the reciprocal basis and the phase deviation equation}

Together with the set $\left\{\mathbf{u}_{1}(t), \ldots, \mathbf{u}_{n}(t)\right\}$ spanning the tangent bundles $T M$ and $T I$, it comes another set $\left\{\mathbf{v}_{1}(t), \ldots, \mathbf{v}_{n}(t)\right\}$, whose elements are defined as the rows of the matrix $\mathbf{V}(t)=\mathbf{U}^{-1}(t)$. Thus by definition, the bi-orthogonality condition

$$
\mathbf{v}_{i}^{T}(t) \mathbf{u}_{j}(t)=\delta_{i j}
$$

holds. It follows that since $\left\{\mathbf{u}_{1}(t), \ldots, \mathbf{u}_{n}(t)\right\}$ is a basis for $\mathbb{R}^{n},\left\{\mathbf{v}_{1}(t), \ldots, \mathbf{v}_{n}(t)\right\}$ is a reciprocal basis, and its elements should be referred to as covectors. The covector $\mathbf{v}_{1}(t)$ is normal to all vectors $\mathbf{u}_{k}(t), k=2, \ldots, n$ and in turn, each covector $\mathbf{v}_{k}(t), k=2 \ldots, n$ is normal to $\mathbf{u}_{1}(t)$. Thus $\mathbf{v}_{1}(t)$ spans the one-dimensional cotangent bundle $N I$ orthogonal to $T I$, while the covectors $\left\{\mathbf{v}_{2}(t), \ldots, \mathbf{v}_{n}(t)\right\}$ span the $(n-1)$-dimensional cotangent bundle $N M$ orthogonal to $T M$.

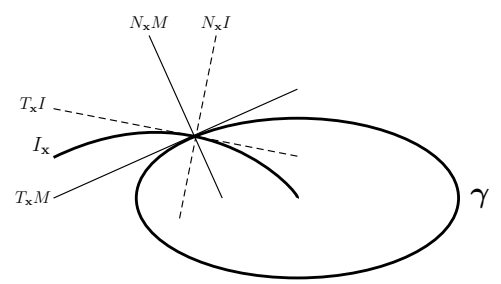

Figure 2: Phase space decomposition of planar nonlinear oscillations. The tangent bundles $T M, T I$ and the cotangent bundles $N M, N I$ at the point $\mathbf{x} \in \boldsymbol{\gamma}$ are shown.

Theorem 3 The covector $\mathbf{v}_{1}(t)$ entering in the phase deviation equation (4) 
and the covector $\mathbf{v}_{2}(t)$ are given by

$$
\begin{aligned}
& \mathbf{v}_{1}(t)=\frac{1}{b(t)}\left(-\alpha(t) \mathbf{f}^{\perp}\left(\mathbf{x}_{0}(t)\right)+\beta(t) \mathbf{f}\left(\mathbf{x}_{0}(t)\right)\right) \\
& \mathbf{v}_{2}(t)=\frac{e^{\mu_{2} t}}{b(t)} \mathbf{f}^{\perp}\left(\mathbf{x}_{0}(t)\right)
\end{aligned}
$$

Proof:the inverse of (30) is

$$
\mathbf{V}(t)=\frac{e^{\mathbf{D} t}}{\beta(t)\|\mathbf{F}(t)\|^{2}}\left[\begin{array}{c}
-\alpha(t) \mathbf{F}^{\perp}(t)^{T}+\beta(t) \mathbf{F}(t)^{T} \\
\mathbf{F}^{\perp}(t)^{T}
\end{array}\right]
$$

from which (35) and (36) stem. We shall now verify that $\mathbf{v}_{1}(t)$ is the unique nontrivial solution of the adjoint problem (5) satisfying the normalization condition (6). From (27) we have $\mathbf{V}(t)=\exp (\mathbf{D} t) \mathbf{V}(0) \mathbf{X}(t, 0)^{-1}$. Let us introduce

$$
\mathbf{Y}(t, 0)=\mathbf{V}(t)^{T} \exp (-\mathbf{D} t) \mathbf{U}(0)^{T}
$$

It is easy to verify that $\mathbf{Y}(t, 0)^{T} \mathbf{X}(t, 0)=\mathbb{1}$. This implies that $\mathbf{Y}(t, 0)$ is a state transition matrix of the adjoint problem. We have

$$
\mathbf{V}(t)^{T}=\mathbf{Y}(t, 0) \mathbf{V}(0) \exp (-\mathbf{D} t)
$$

which implies $\mathbf{v}_{1}(t)=\mathbf{Y}(t, 0) \mathbf{v}_{1}(0)$. By taking the derivative we get

$$
\dot{\mathbf{v}}_{1}(t)=\dot{\mathbf{Y}}(t, 0) \mathbf{v}_{1}(0)=-\mathbf{A}^{T}(t) \mathbf{Y}(t, 0) \mathbf{v}_{1}(0)=-\mathbf{A}^{T}(t) \mathbf{v}_{1}(t)
$$

that is, $\mathbf{v}_{1}(t)$ is a solution of the adjoint problem. Finally, by definition $\mathbf{V}(t) \mathbf{U}(t)=$ $\mathbb{1}$, that is $\mathbf{v}_{i}(t)^{T} \mathbf{u}_{j}(t)=\delta_{i j}$, which implies $\mathbf{v}_{1}(t)^{T} \mathbf{F}(t)=1$ as required.

The bi-orthogonality condition (34) implies that at each time instant the covector $\mathbf{v}_{1}(t)$ is parallel to the gradient of the isochron. Thus $\mathbf{v}_{1}(t)$ identifies the direction along which the isochron is most sensitive to the perturbations. At glance, eq. (4) and (35) may look rather difficult to use in practical application. However, one can take advantage of the periodicity of $\mathbf{v}_{1}(t)$ and consider its Fourier series [10, 11]

$$
\mathbf{v}_{1}(t)=\sum_{k=-\infty}^{+\infty} V_{k} e^{i k \omega t}
$$

In this case, eq. (35) can be a practical tool to compute the spectral coefficients $V_{k}$.

Figure 3 shows the application of the method to the van der Pol oscillator. The vectors tangent and those transversal to the cycle represent the vectors $u_{1}$ and $u_{2}$ respectively, at different time instants. The filled circles represent the initial conditions of trajectories starting on the plane spanned by $u_{2}(\bar{t})$, and thus tangent to the isochron based at $\gamma(\bar{t})$. The empty circles represent the same trajectories after a long enough transient. All trajectories are very close to the limit cycle and share the same phase. The filled and the empty squares are the analogues for trajectories with initial condition outside the isochron. In this case the final points have different phases. 


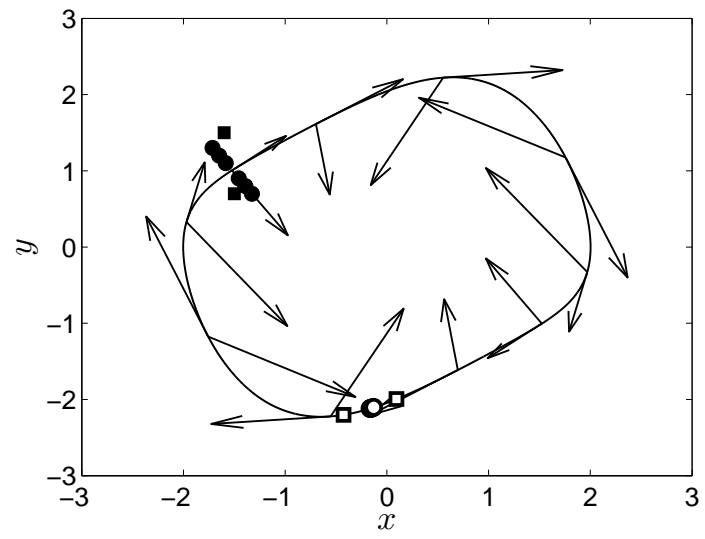

Figure 3: Application of the method to the van der Pol oscillator. The explanation is given in the text.

\section{On the validity of an orthogonal decomposi- tion}

From eqs. (13) and (14) we recognize that, in general, $T M$ and $T I$ are not orthogonal spaces. This explains why the original conjecture proposed in [5] to decompose the perturbation into orthogonal components is, in general, incorrect. However, it turns out that for a class of oscillator the orthogonal decomposition is appropriate.

Theorem 4 For all those oscillators such that $\mathbf{f}^{T}\left(\mathbf{x}_{0}(t)\right)\left(\mathbf{A}(t)+\mathbf{A}^{T}(t)\right) \mathbf{f}^{\perp}\left(\mathbf{x}_{0}(t)\right)=$ 0 , the perturbation can be decomposed into two orthogonal components.

Proof: we have

$$
\mathbf{u}_{1}(t)^{T} \mathbf{u}_{2}(t)=\alpha(t)\|\mathbf{F}(t)\|^{2} e^{\mu_{2} t}
$$

which can be identically null if and only if $\alpha(t)=0$ for all $t \in \mathbb{R}$. By eq. (15) this implies $a(t)=a(T) /(1-b(T))$, that is, $a(t)$ must be a constant. Since $\mathbf{X}(T, 0)$ is regular, we have $b(T) \neq 0$, and it stems that $a(t)$ must be null for all $t$. This condition is obviously satisfied if and only if $\mathbf{F}(t)^{T}\left(\mathbf{A}(t)+\mathbf{A}^{T}(t)\right) \mathbf{F}^{\perp}(t)=0$.

We shall now show that the condition above is equivalent to a classical result about the admissibility of an orthogonal decomposition. Some preliminary considerations are needing. The isochrons represent the leaves of an invariant foliation of the oscillator stable manifold [15]. A classical result in nonlinear control theory states that if a given vector field is tangent to a foliation, and another vector filed preserves this foliation, then the Lie bracket of the vector fields 
is tangent to the foliation [18. It stems that a necessary and sufficient condition for $\mathbf{f}^{\perp}$ to generate an invariant foliation of the oscillator's stable manifold is

$$
\left[\mathbf{f}, \mathbf{f}^{\perp}\right]\left(\mathbf{x}_{0}(t)\right)=c \mathbf{f}^{\perp}\left(\mathbf{x}_{0}(t)\right)
$$

where $[\cdot, \cdot]$ represents the Lie bracket and $c \in \mathbb{R}$.

Theorem 5 The condition

$$
\mathbf{f}\left(\mathbf{x}_{0}(t)\right)\left(\mathbf{A}(t)+\mathbf{A}^{T}(t)\right) \mathbf{f}^{\perp}\left(\mathbf{x}_{0}(t)\right)=0
$$

is equivalent to $\left[\mathbf{f}, \mathbf{f}^{\perp}\right]\left(\mathbf{x}_{0}(t)\right)=c \mathbf{f}^{\perp}\left(\mathbf{x}_{0}(t)\right)$

Proof: we shall prove that the first condition implies the second. The converse can be proved in the same way. For the sake of simplicity we simply write $\mathbf{f}, \mathbf{f}^{\perp}$ and $\mathbf{A}$ omitting the arguments $\mathbf{x}_{0}(t)$ and $t$. By hypothesis $\left(\mathbf{A}+\mathbf{A}^{T}\right) \mathbf{f}^{\perp}=c^{*} \mathbf{f}^{\perp}$, for some $c^{*} \in \mathbb{R}$. We recall that the Lie bracket of vector fields is defined by $[\mathbf{f}, \mathbf{g}]=\mathbf{D g} \mathbf{f}-\mathbf{D f} \mathbf{g}$ where $\mathbf{D f}$ and $\mathbf{D g}$ are Jacobian matrices. A routine calculation shows that

$$
\left[\mathbf{f}, \mathbf{f}^{\perp}\right]=\left((\nabla \cdot \mathbf{f}) \mathbf{f}^{\perp}-\left(\mathbf{A}+\mathbf{A}^{T}\right) \mathbf{f}^{\perp}\right)=\left((\nabla \cdot \mathbf{f})-c^{*}\right) \mathbf{f}^{\perp}
$$

and by setting $\left(\nabla \cdot \mathbf{f}-c^{*}\right)=c$ the proof is completed.

\section{Conclusions}

In this brief we have presented a rigorous decomposition for the phase space of weakly perturbed planar nonlinear oscillators. We have derived analytical formulas for the basis spanning the directions onto which project the perturbations. We have also derived analytical formulas for the complementary basis formed by the associated covectors. These equations have been derived using a classical result on the integration of planar homogenous differential equations, the Diliberto's theorem, they are rigorous and expressed in terms of the unperturbed limit cycle, only. Since they are exact, they can be used as benchmarks to test the accuracy of numerical methods. Our results allow to write the phase deviation equation and the Fokker-Planck equation derived in [6] in a closed analytical form. These equations can be exploited to investigate the synchronization of a nonlinear oscillator with external signals and the influence of noise on the oscillator's output spectrum. This will be the topic of future works.

\section{References}

[1] M. Lax, "Classical noise vs. noise in self sustained oscillators," Phys. Rev., vol. 160, pp. 290-307, 1967. 
[2] M. Okumura, T. Sugawara, and H. Tanimoto, "An effcient small signal frequency analysis method for nonlinear circuits with two frequency excitations," IEEE Trans. Comput.-Aided Design Integr. Circuits Syst., vol. 9, no. 3, pp. 225-235, March 1990.

[3] F. K. Kärtner, "Determination of the correlation spectrum of oscillators with low noise," IEEE Trans. Microw. Theory Techn., vol. 37, no. 1, pp. 90-101, January 1989.

[4] - "Analysis of white and $f^{-\alpha}$ noise in oscillators," Int. J. Circuit Theory Appl., vol. 18, pp. 485-519, 1990.

[5] A. Hajimiri and T. H. Lee, "A general theory of phase noise in electrical oscillators," IEEE J. Solid-State Circuits, vol. 33, no. 2, pp. 179-194, February 1998.

[6] A. Demir, A. Mehrotra, and J. Roychowdhury, "Phase noise in oscillators: A unifying theory and numerical methods for characterization," IEEE Trans. Circuits Syst. I, vol. 47, no. 5, pp. 655-674, May 2000.

[7] A. Demir and J. Roychowdhury, "A reliable and efficient procedure for oscillator PPV computation, with phase noise macromodeling applications," IEEE Trans. Comput.-Aided Design Integr. Circuits Syst., vol. 22, no. 2, pp. 188-197, February 2003.

[8] X. Lai and J. Roychowdhury, "Capturing oscillator injection locking via nonlinear phase-domain macromodels," IEEE Trans. Microw. Theory Techn., vol. 52, no. 9, pp. 2251-2261, September 2004.

[9] P. Maffezzoni, "Analysis of oscillator injection locking through phasedomain impulse-response," IEEE Trans. Circuits Syst. I, vol. 55, no. 5, pp. 1297-1305, June 2008.

[10] - "Synchronization analysis of two weakly coupled oscillators through a PPV macromodel," IEEE Trans. Circuits Syst. I, vol. 57, no. 3, pp. 654-663, March 2010.

[11] P. Maffezzoni and D. D'Amore, "Evaluating pulling effects in oscillators due to small-signal injection," IEEE Trans. Comput.-Aided Design Integr. Circuits Syst., vol. 28, no. 1, pp. 22-31, January 2009.

[12] A. Demir, "Phase noise and timing jitter in oscillators with colored-noise sources," IEEE Trans. Circuits Syst. I, vol. 49, no. 12, pp. 1782-1791, December 2002.

[13] F. L. Traversa and F. Bonani, "Oscillator noise: a nonlinear perturbative theory including orbital fluctuations and phase-orbital correlation," IEEE Trans. Circuits Syst. I, vol. 58, no. 1, pp. 2485-2497, October 2011. 
[14] S. N. Chow and H. M. Zhou, "An analysis of phase noise and Fokker-Planck equations," J Differ Equations, vol. 234, pp. 391-411, 2007.

[15] T. Djurhuus, V. Krozer, J. Vidkjær, and T. K. Johansen, "Oscillators phase noise: A geometrical approach," IEEE Trans. Circuits Syst. I, vol. 56, no. 7, pp. 1373-1382, July 2009.

[16] A. Brambilla, P. Maffezzoni, and G. Storti-Gajani, "Computation of period sensitivity functions for the simulation of phase noise in oscillators," IEEE Trans. Circuits Syst. I, vol. 52, no. 4, pp. 681-694, April 2005.

[17] C. Chicone, "Bifurcations of Nonlinear Oscillations and Frequency Entrainment Near Resonance," SIAM J. Math. Anal., vol. 23, no. 6, pp. 1577-1608, 1992.

[18] B. Jakubczyck, Introduction to geometric nonlinear control; controllability and Lie bracket, ser. Mathematical control theory, Part 1, 2, ICTP Lectures Notes. Trieste, Italy: Abdus Salam Int. Cent. Theoret. Phys., 2001. 